\title{
Article \\ Evaluation of Smart Chromatic Technology for a Single-Shade Dental Polymer Resin: An In Vitro Study
}

\author{
Eman M. AlHamdan ${ }^{1}$, Amr Bashiri ${ }^{2}$, Faisal Alnashmi ${ }^{2}$, Samar Al-Saleh ${ }^{1}$, Khalid Al-shahrani ${ }^{2}$, \\ Saad Al-shahrani ${ }^{2}$, Abdullah Alsharani ${ }^{1}$, Khaled M. Alzahrani ${ }^{3}$, Firas K. Alqarawi ${ }^{4}$, Fahim Vohra ${ }^{5, *(D)}$ \\ and Tariq Abduljabbar 5 (D)
}

Citation: AlHamdan, E.M.; Bashiri, A.; Alnashmi, F.; Al-Saleh, S.; Al-shahrani, K.; Al-shahrani, S.; Alsharani, A.; Alzahrani, K.M.; Alqarawi, F.K.; Vohra, F.; et al. Evaluation of Smart Chromatic Technology for a Single-Shade Dental Polymer Resin: An In Vitro Study. Appl. Sci. 2021, 11, 10108. https:// doi.org/10.3390/app112110108

Academic Editors: Paola Gandini and Andrea Scribante

Received: 13 September 2021

Accepted: 22 October 2021

Published: 28 October 2021

Publisher's Note: MDPI stays neutra with regard to jurisdictional claims in published maps and institutional affiliations.

Copyright: (c) 2021 by the authors. Licensee MDPI, Basel, Switzerland. This article is an open access article distributed under the terms and conditions of the Creative Commons Attribution (CC BY) license (https:/ / creativecommons.org/licenses/by/ $4.0 /)$.
1 Department of Prosthetic Dental Science, College of Dentistry, King Saud University, P.O. Box 21069, Riyadh 11475, Saudi Arabia; ealhamdan@ksu.edu.sa (E.M.A.); salsaleh@ksu.edu.sa (S.A.-S.); asalshahrani@ksu.edu.sa (A.A.)

2 Intern, Department of General Dentistry, College of Dentistry, King Saud University, Riyadh 11545, Saudi Arabia; afbashiri@gmail.com (A.B.); fyalnashmi@hotmail.com (F.A.); khlo0ode1995@gmail.com (K.A.-s.); s.moflehh@gmail.com (S.A.-s.)

3 Department of Prosthetic Dental Sciences, College of Dentistry, Prince Sattam Bin AbdulAziz University, Alkharj 11942, Saudi Arabia; dr_kmq@hotmail.com

4 Department of Substitutive Dental Sciences, College of Dentistry, Imam Abdulrahman Bin Faisal University, Dammam 34212, Saudi Arabia; fkalqarawi@iau.edu.sa

5 Department of Prosthetic Dental Science, College of Dentistry, King Saud University, Research Chair for Biological Research in Dental Health, Riyadh 11545, Saudi Arabia; tajabbar@ksu.edu.sa

* Correspondence: fvohra@ksu.edu.sa; Tel.: +966-143755444

Abstract: The aim of the study was to assess the color matching ability and color stability of a single-shade resin-based composite (Omnichroma-OM) in comparison to a conventional dental composite. One hundred and sixty lower molar acrylic teeth in four different shades (B1, B2, A3, and C3) were prepared with class I cavities. Eighty teeth were divided into two groups based on the two resin composite materials (OM and Filtek Z-350 (FT)) for cavity restoration. Three groups were included in the color matching assessment, namely Gp 1-FT, Gp 2-OM-light cured (C), and Gp 3-OM-uncured (UC) groups. The color assessment was performed prior to cavity restoration and after cavity filling and before and after light curing to detect color matching. The 80 remaining teeth were restored with OM $(n=40)$ and FT $(n=40)$; half $(n=40)$ were submerged in coffee and the other half $(n=40)$ were submerged in cola for 2 weeks. The color stability was assessed by calculating material color (spectrophotometry) changes $(\Delta \mathrm{E})$ before and after staining in immersion medium. The means and standard deviations of the $\Delta \mathrm{E}$ values relating to color matching and color stability (stain resistance) among the study groups were analyzed using ANOVA and Tukey's post hoc test. FT showed significantly better color matching (lower $\Delta \mathrm{E}$ ) in comparison to OM-UC specimens for shades B1, B2, and A3 ( $p<0.01)$. Regarding the color stability in cola, the FT samples showed significantly lower $\Delta \mathrm{E}$ values (indicating better color stability) compared to OM samples for all four shades $(p<0.01)$. The $\Delta \mathrm{E}$ values of OM and FT samples in coffee were comparable $(p>0.01)$, showing comparable color stability. The color matching ability of the single-shade resin composite (OM) was influenced by the tooth color. The conventional resin composite showed better color matching than the single-shade composite (OM). The color stability of the single-shade resin composite (OM) was dependent on the staining medium. The OM composite showed more compromised color stability than the conventional resin composite in cola and comparable color stability to conventional resin composite in coffee.

Keywords: resin composite; Omnichroma; color stability; color matching

\section{Introduction}

One of the most admired features of restorative materials is their aesthetics. In recent times, the search for restorative materials in ideal shades for each patient has been 
a difficult task [1]. Among the many restorative materials, resin-based materials have displayed some of the best outcomes clinically. Resin composites have been proven to be excellent tooth replacement materials and a great choice for a plethora of clinical indications due to their good adhesive bonding, aesthetics, translucency, mechanical properties, and biocompatibility $[2,3]$.

Many dentists struggle when matching the shade of a tooth with that of the desired restorative material. A demarcation always remains between the two entities, which is known as the blending effect (BE) [4]. In the literature, this is explained as an evident demarcation of the restoration from the tooth structure under isolation [5]. However, the blending effect in shaded resin composites is influenced by the restoration size, as the $\mathrm{BE}$ for fillings increases with decreasing restoration size and increasing filling material translucency. Moreover, BE increases when the color difference between the restoration and the surrounding walls decreases [5,6]. To avoid this problem, many dentists use a mixture of resin composite shades, employing a layering technique.

Moreover, the optical properties also play an important role in the appearance of a restoration. The illumination, translucency, opacity, light scattering, gloss, and human sensitivity influence the overall perception of tooth color $[7,8]$. Teeth are made up of layers called enamel and dentin, which differ in terms of their structural characteristics and optical properties [8]. Both exhibit different light wave characteristics, which reflect upon the appearance of the teeth [9]. Due to its highly mineralized prismatic structure, low organic content, and small amount of water, higher transmission of light occurs with enamel than dentin. In contrast, dentin has a lower mineral content, with an organic tubular structure, low translucency, and a high water content [10].

The light reflection and transmission of modern resin-based restorative materials used for tooth structure repair are influenced by the materials' organic and inorganic constituents and proportions [11,12]. One ideal standard is to develop advanced restorative materials with properties similar to the tooth structure. The aim is to simulate the natural tooth in terms of color, translucency, and surface texture in order to achieve long-term color stability. In recent times, resin-based materials have shown improved physical, mechanical, and optical properties. However, resin composites are low in biomimetic potential due to their non-crystalline structures, low translucency, and absence or presence of fluorescence $[13,14]$. These characteristics and others make color matching between the resin composite and the tooth structure much more difficult to achieve $[15,16]$.

In recent times, a novel single-shade resin restorative composite (Omnichroma (OM)) was designed using smart chromatic technology, with $260 \mathrm{~nm}$ spherical fillers and a similar appearance as the surrounding tooth structure [17]. OM is available as a single-color universal composite and was designed for use in most direct restorative clinical cases. Its wide color matching ability eliminates the need for a shade assessment procedure and reduces the composite inventory, allowing clinicians to minimize chair time, the wastage of unused composite shades, and to reduce reliance on shade matching procedures. However, there is limited evidence on the success of its color matching ability in dental restorations. In a recent study on OM, Evans (2020) suggested that the shade difference between the composite $(\mathrm{OM})$ and the tooth decreases as the tooth becomes brighter [15]. In addition, $\mathrm{OM}$ has shown compromised aesthetic outcomes when employed in direct restorations $[17,18]$. Therefore, it is critical to ascertain the color matching ability and color stability of OM in comparison to conventional resin-based restorative materials. It is hypothesized that the color matching ability and color stability of the single-shade resin composite (OM) will be comparable to the conventional resin-based composite restorative material. Therefore, in the present study we aim to assess the color matching ability and color stability of a singleshade resin composite (OM) material in comparison to a conventional resin composite. 


\section{Materials and Methods}

\subsection{Specimen Preparation}

Eighty lower molar acrylic teeth of four different shades (B1, B2, A3, and C3) were prepared using standardized, simulated class I preparations, a high-speed hand piece (Henry Schein, Melville, NY, USA) and number 245 carbide burs (Henry Schein, Melville, NY, USA). The sample dimensions were $2 \mathrm{~mm}$ in depth and $4 \mathrm{~mm}$ in diameter (Figure 1). The prepared cavities were rinsed with water and air-dried. All tooth preparations were performed by a single operator $(\mathrm{AB})$. Based on the four different tooth shades, the prepared teeth were divided into 4 groups of 20 teeth each (B1, B2, A3, and C3).

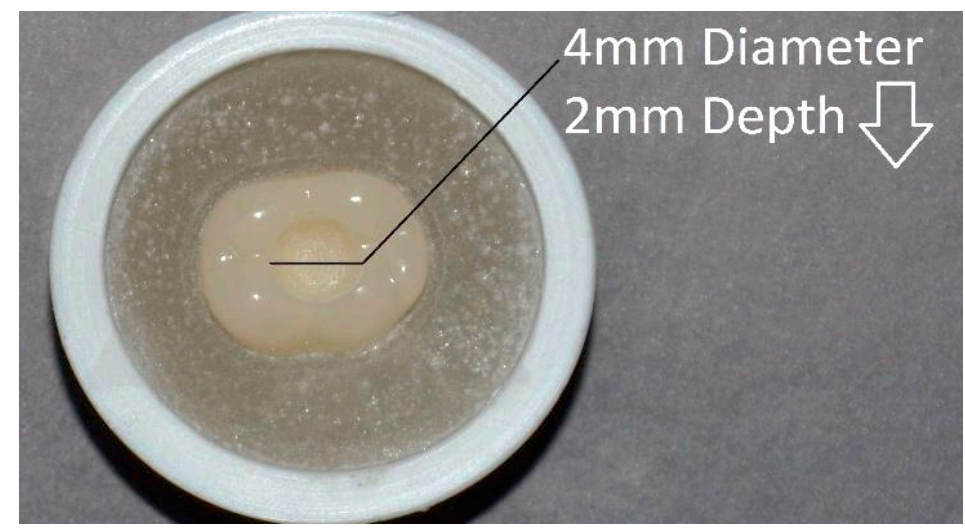

Figure 1. Prepared acrylic molar tooth used for restoration.

\subsection{Specimen Restoration and Study Groups (Color Matching)}

The prepared teeth were further divided into two groups based on the type of restorative materials used for restoring class 1 cavities. Half of the prepared teeth (40) were restored with a single-shade (OM) (single-shade universal composite, Tokuyama Dental, Encinitas, CA, USA) (test group) and the other half were restored with a conventional resinbased composite (Filtek Z-350 (FT), 3M technology, Minnesota, MN, USA) (control group). Based on the combinations of tooth shades, restorative materials, and photopolymerization, the specimens were divided into 3 groups.

Group 1. Filtek (FT) - Teeth restored (photopolymerized/light cured) with Filtek Z-350 resin-based composite. This group consisted of four subgroups with four different acrylic tooth shades (B1, B2, A3, and C3). All teeth were restored with the corresponding color resin material (Filtek).

Group 2. OM-C-Teeth restored (photopolymerized/light cured) with OM resinbased composite. This group consisted of four subgroups with four different acrylic tooth shades (B1, B2, A3, and C3). All teeth were restored with the same shade of OM resin material.

Group 3. OM-UC-Teeth restored (unpolymerized/uncured) with OM resin-based composite (polymerization of resin was not performed). This group consisted of four subgroups with four different acrylic tooth shades (B1, B2, A3, and C3). All teeth were restored with the same shade of OM resin material.

The same restored tooth specimens were included in groups 2 and 3. Specimens in group 3 were initially uncured (OM-UC) and assessed for color or shade, then were later photopolymerized (OM-C) prior to post-curing color assessment.

Cleaned cavities were air-dried and restored in two increments measuring $2 \mathrm{~mm}$ deep and $2 \mathrm{~mm}$ wide for both materials. Resin-based materials were dispensed in a dark container and packed with a plastic instrument and condenser. The restoration material was standardized for each cavity, as the cavity dimensions were similar and the material placement teeth were same for all specimens. In addition, a single operator performed all restorations (KA). Excess material was removed and margins were adapted with a plastic instrument, while the occlusal surface was flat. A mylar strip was covered with a glass 
slide and the restorative material was photocured for $40 \mathrm{~s}$ from the occlusal aspect. A $20 \mathrm{~s}$ polymerization exposure process was also performed on buccal and lingual surfaces. Polymerization was performed using an LED light curing unit (3M Elipar Deep Cure-S LED, Minnesota, MN, USA) with a light intensity of $400 \mathrm{~mW} / \mathrm{cm}^{2}$ in a uniform, continuous curing mode (Figure 2).

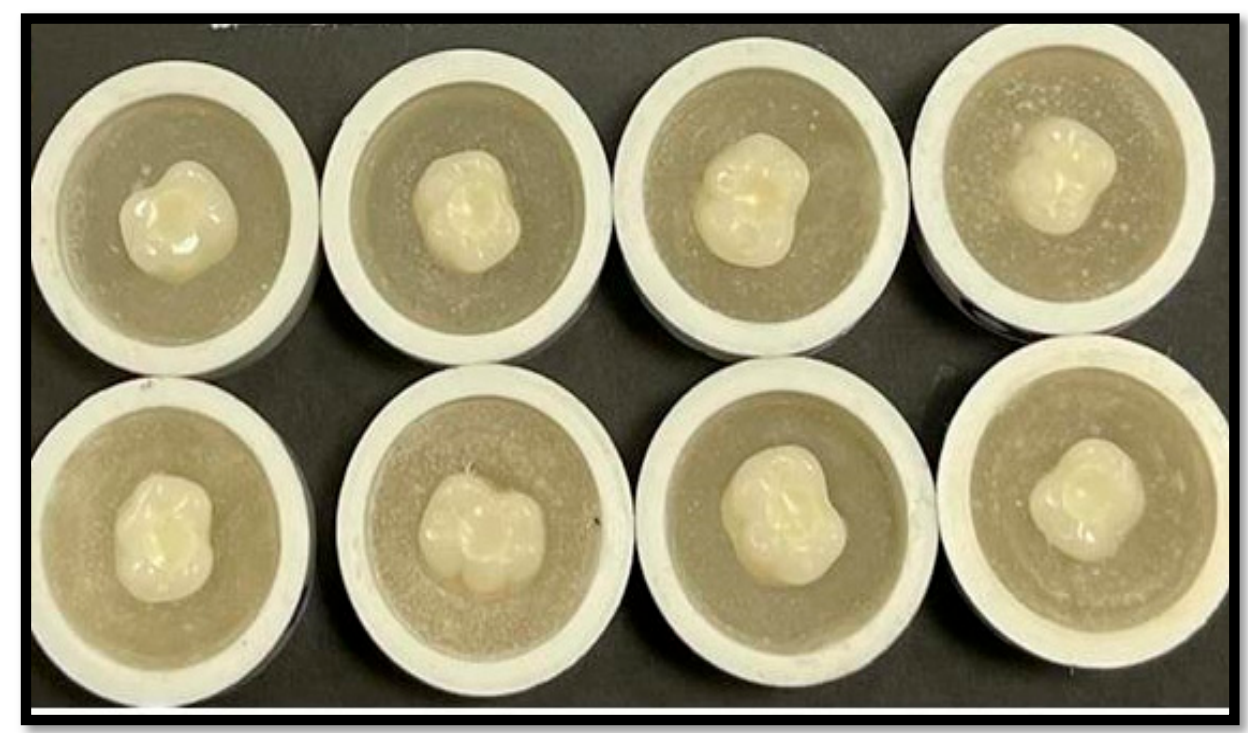

Figure 2. Color matching assessment of restored tooth specimens from the different study groups.

Baseline tooth colors were assessed for different shaded acrylic teeth on the occlusal surface prior to restoration with different resin-based materials. For group 1 (FT), color was assessed after restoration in four different colored teeth. However, in teeth restored with OM, the color was assessed prior to light curing (group 3-OM-UC) and after light curing (group 2-OM-C).

\subsection{Specimen Preparation (Color Stability)}

One hundred and sixty lower molar acrylic teeth of four different shades (B1, B2, A3, and C3) were prepared with standardized simulated class I preparations using a high-speed hand piece (Henry Schein, Melville, NY, USA) and number 245 carbide burs (Henry Schein, Melville, NY, USA). The preparation dimensions were $2 \mathrm{~mm}$ in depth and $4 \mathrm{~mm}$ in diameter. The prepared cavities were rinsed with water and air-dried. All tooth preparations were performed by a single operator (AB). Based on the four different teeth shades, the prepared teeth were divided into 4 groups of 40 teeth each (B1, B2, A3, and C3).

Half of the prepared teeth $(n=80)$ were restored with a single-shade OM (single-shade universal composite, Tokuyama Dental, Encinitas, CA, USA) (test group) and the other half $(n=80)$ were restored with a conventional resin-based composite (Filtek Z-350 (FT), $3 \mathrm{M}$ technology, Minnesota, MN, USA) (control group). Based on the combinations of tooth shades, restorative materials, and staining mediums, the specimens were divided into 4 groups.

Group 1. Filtek-Cola $(n=40)$ : Acrylic teeth restored with Filtek Z-350 resin-based composite were immersed in a carbonated drink (Pepsi, Pepsi Co., Harrison, NY, USA) for 2 weeks. This group consisted of four subgroups with four different acrylic tooth shades (B1, B2, A3, and C3). All teeth were restored with the corresponding color resin material (Filtek).

Group 2. Filtek-Coffee $(n=40)$ : Acrylic teeth restored with Filtek Z-350 resin-based composite were immersed in coffee (Nestlé USA, Inc., Glendale, CA, USA) for 2 weeks. This group consisted of four subgroups with four different acrylic tooth shades (B1, B2, A3, and C3). All teeth were restored with the corresponding color resin material (Filtek). 
Group 3. OM-Cola $(n=40)$ : Acrylic teeth restored with OM resin-based composite were immersed in carbonated drink (Pepsi, Pepsi Co., Harrison, NY, USA) for 2 weeks. This group consisted of four subgroups with four different acrylic tooth shades (B1, B2, A3, and C3). All teeth were restored with the same shade of OM resin material.

Group 4. OM-Coffee $(n=40)$ : Acrylic teeth restored with OM resin-based composite were immersed in coffee (Nestlé USA, Inc., Glendale, CA, USA) for 2 weeks. This group consisted of four subgroups with four different acrylic tooth shades (B1, B2, A3, and C3). All teeth were restored with the same shade of OM resin material.

Specimens in groups 1 and 3 were immersed in a carbonated drink, which was replaced every $24 \mathrm{~h}$ for 2 weeks [8]. To prepare the coffee solution, 25 grams of coffee (Nestlé USA, Inc., Glendale, CA, USA) was poured into 250 milliliters of water at $100{ }^{\circ} \mathrm{C}$. The solution was stirred for $10 \mathrm{~min}$ and filtered. The coffee solution was replaced every $24 \mathrm{~h}$. Each specimen was assessed for baseline color after $24 \mathrm{~h}$ of restoration, prior to immersion in the stain solutions (carbonated drink and coffee). After removal from the stain solution, specimens were washed under running water for 5 min and dried. All specimens were assessed for color change after staining.

\subsection{Color Assessment}

The specimen color or shade was assessed using a spectrophotometer (Vita Easyshade ${ }^{\circledR} \mathrm{V}$ Compact Vita, Zahnfabrik, Bad Sackingen, Germany) to evaluate the color matching ability and color stability (staining resistance) of the single-shade OM resin in comparison to a conventional resin composite. A positioning jig was used to place the specimens in a reproducible manner. The photometer was calibrated in accordance with the National Institute of Standards and Technology (NIST) tiles. The setting at which the tests were run included a $10 \mathrm{~nm}$ wavelength interval, 360 to $750 \mathrm{~nm}$ spectral range, and $45^{\circ}$ reflectance angle. The background was black and an average of three scans were performed for each specimen. The CIELAB color system was employed, comprising $L^{*}, a^{*}$, and $b^{*}$ axes identification. Here, "L " ranged from 0 to 100 (brightness to darkness), the "a " axis represented red to green colors ( 90 to 70 range value), and the " $\mathrm{b}$ " axis represented yellow to blue colors (coordinate value range from: 80 to 100). Values for $\mathrm{L}^{*}, \mathrm{a}^{*}$, and $\mathrm{b}^{*}$ were compared from baseline (tooth color) to post-restoration for the color matching assessment. For the color stability assessment (stain resistance), $\mathrm{L}^{*}, \mathrm{a}^{*}$, and $\mathrm{b}^{*}$ differences $(\Delta)$ were calculated before and after stain solution immersion. The color change $\Delta \mathrm{E}$ was calculated using the following equation:

$$
\Delta \mathrm{E}=\left(\Delta \mathrm{L}^{2}+\Delta \mathrm{a}^{2}+\Delta \mathrm{b}^{2}\right)^{1 / 2}
$$

\subsection{Statistical Analysis}

Data were tabulated in Excel and were statistically analyzed with Statistical Package for Social Sciences (SPSS Inc, 23, IBM, Chicago, IL, USA). The $\triangle \mathrm{E}$ obtained for the color matching test was analyzed with ANOVA and individual groups were compared using Tukey's post hoc test. Similarly for the color stability assessment, $\Delta \mathrm{E}$ (color difference) values among the restorative materials and staining medium groups were compared using ANOVA and a post hoc test. Here, $p$ values of $\leq 0.01$ were considered significant.

\section{Results}

\subsection{Color Matching Ability}

The present study evaluated the color matching ability of single-shade OM resin against a standard restorative material, Filtek-Z 350 (FT). The one-way ANOVA showed significant differences among the study groups $(p<0.01)$. The $\Delta \mathrm{E}$ (color difference) values for the four shades of the two composites were compared (Table 1) for shade matching within each material. The highest mean $\triangle \mathrm{E}$ among the study groups was in shade $\mathrm{B} 1$ (17.806 \pm 1.8722$)$ for OM, while the lowest was in FT shade for A3 (2.47 \pm 0.6225$)$. Moreover, in the FT group, the highest $\Delta \mathrm{E}$ among the acrylic teeth and restoration samples was 
for B1 $(9.0956 \pm 0.7721)$, while the lowest was for shade A3 (2.47 \pm 0.6225$)$. A similar pattern was observed among the OM groups, with shade $\mathrm{B} 1$ showing the highest $\triangle \mathrm{E}$ and shade $\mathrm{C} 3$ displaying the lowest $\Delta \mathrm{E}$. Furthermore, a comparison between the 4 shades demonstrated significant differences among the 2 materials, Filtek and OM $(p<0.01)$ (Table 1). Overall, the shade and color matching ability levels of FT, OM-C, and OM-UC were significantly different. A statistical comparison among these groups for a specific shade showed significantly different $p$ values (B1: <0.0001; B2: <0.0001; A3: <0.0001; C3: 0.001) (Table 1).

Table 1. Mean and standard deviation of color differences $(\Delta \mathrm{E})$ among study groups and statistical comparisons.

\begin{tabular}{|c|c|c|c|c|c|}
\hline \multirow{3}{*}{ Resin Materials } & \multicolumn{4}{|c|}{ Shade } & \multirow{3}{*}{$p$ Value * } \\
\hline & B1 & B2 & A3 & $\mathrm{C} 3$ & \\
\hline & \multicolumn{4}{|c|}{ Mean (SD) } & \\
\hline FT & $\begin{array}{c}4.0956 \\
(0.7721)\end{array}$ & $\begin{array}{c}2.99 \\
(0.9316)\end{array}$ & $\begin{array}{c}2.47 \\
(0.6225)\end{array}$ & $\begin{array}{l}3.6175 \\
(1.283)\end{array}$ & $<0.00001$ \\
\hline OM-C & $\begin{array}{c}7.265 \\
(0.6096)\end{array}$ & $\begin{array}{c}3.4186 \\
(0.3753)\end{array}$ & $\begin{array}{c}3.2425 \\
(0.5662)\end{array}$ & $\begin{array}{c}2.3938 \\
(0.3433)\end{array}$ & $<0.00001$ \\
\hline OM-UC & $\begin{array}{c}17.806 \\
(1.8722)\end{array}$ & $\begin{array}{c}5.0657 \\
(0.6068)\end{array}$ & $\begin{array}{c}4.5638 \\
(0.4122)\end{array}$ & $\begin{array}{c}4.4225 \\
(1.0452)\end{array}$ & $<0.00001$ \\
\hline$p$ value * & $<0.00001$ & 0.000039 & $<0.00001$ & 0.001704 & \\
\hline
\end{tabular}

Table 2 presents a comparison of $p$ values among the study groups for color matching. Comparing FT (control) with OM-C showed significantly better color matching for FT for shades BI ( $p$ 0.008) and A3 ( $p$ 0.004). However, color matching among FT and OM-C was comparable for shades B2 ( $p$ 0.478) and C3 ( $p$ 0.051). FT showed better color matching than OM-C for groups B1, B2, and A3; however, OM-C showed better color matching than FT for shade C3 (Table 1). OM-C showed significantly better color matching (lower $\Delta \mathrm{E}$ ) in comparison to OM-UC specimens for all tooth shades $(p<0.01)$. FT showed significantly better color matching (lower $\Delta \mathrm{E}$ ) in comparison to OM-UC specimens for shades B1, B2, and A3 $(p<0.01)$; however, shade C3 showed comparable color matching ability for FT and OM-UC $(p>0.01)$.

Table 2. Comparison of $p$-values $(\Delta \mathrm{E})$ for the color matching ability of the restorative resin materials.

\begin{tabular}{ccccc}
\hline \multirow{2}{*}{ Comparing Study Groups } & B1 & \multicolumn{4}{c}{ B2 } & A3 & C3 \\
\cline { 2 - 5 } & \multicolumn{5}{c}{$p$-Value $^{*}$} \\
\cline { 2 - 5 } & 0.00854 & 0.478 & 0.00461 & 0.05128 \\
\hline FT-OM C & 0.00000 & 0.00005 & 0.00000 & 0.24759 \\
\hline FT-OM UC & 0.00000 & 0.00062 & 0.00022 & 0.00124 \\
\hline OMC-OM UC &
\end{tabular}

Note: * Tukey's post hoc test; FT-Filtek; OM-Omnichroma; C—cured/polymerized; UC—uncured/unpolymerized.

\subsection{Color Stability}

The color stability of the restorative materials (FT and OM) was assessed using two commonly used beverages: cola and coffee. The lowest $\Delta \mathrm{E}$ values were observed for FT samples in FT-cola (mean $\Delta \mathrm{E} 1.30-1.81$ ), while the highest $\Delta \mathrm{E}$ values were observed in FT-coffee samples (8.83-13.20). Among the cola samples, FT samples showed significantly lower $\Delta \mathrm{E}$ compared to OM samples for all four shades (B1,B2, A3, C3) $(p<0.01)$ (Table 3). Having different shades of teeth had no influence on the color stability of samples stained with cola. Therefore, OM showed lower color stability and stain resistance than FT with cola immersion. Furthermore, after the submersion into coffee, FT showed higher $\Delta \mathrm{E}$ than 
OM for shades B1 (FT, $13.20 \pm 2.2-$ OM 9.55 \pm 0.79), B2 (FT-11.75 \pm 1.77; OM-9.06 \pm 0.53$)$, and A3 (FT, $10.71 \pm 0.82-\mathrm{OM} 9.63 \pm 1.30)$. The OM specimen in coffee showed significantly lower color change (better stain resistance) than FT samples for shade B1 $(p=0.00006)$ (Table 3). However, the $\Delta \mathrm{E}$ values of $\mathrm{OM}$ and FT samples in coffee were comparable ( $p>0.01$ ) for shades B2, A3, and C3 (Table 3). The differences between shades did not have a significant influence on $\Delta \mathrm{E}$ values in OM-coffee specimens, although FT-coffee samples showed significantly different $\Delta \mathrm{E}$ values $(p 0.0001)$ due to the different tooth shades. Figure 3 shows the samples after staining medium immersion.

Table 3. Mean color differences $(\Delta \mathrm{E})$ and standard deviations for the four shades of the two composite materials regarding color stability.

\begin{tabular}{|c|c|c|c|c|c|}
\hline \multirow{3}{*}{ Comparison among Groups } & \multicolumn{4}{|c|}{ Shade } & \multirow{3}{*}{$p$ Value ${ }^{\S}$} \\
\hline & B1 & B2 & A3 & $\mathrm{C} 3$ & \\
\hline & \multicolumn{4}{|c|}{$\begin{array}{l}\text { Mean } \\
\text { (SD) }\end{array}$} & \\
\hline FT-Cola & $\begin{array}{c}1.68 \\
(0.40)\end{array}$ & $\begin{array}{c}1.33 \\
(0.12)\end{array}$ & $\begin{array}{c}1.3 \\
(0.4)\end{array}$ & $\begin{array}{c}1.81 \\
(0.54)\end{array}$ & 0.21552 \\
\hline OM-Cola & $\begin{array}{c}4.72 \\
(0.40)\end{array}$ & $\begin{array}{c}4.73 \\
(0.65)\end{array}$ & $\begin{array}{c}4.51 \\
(0.47)\end{array}$ & $\begin{array}{c}5.54 \\
(0.25)\end{array}$ & 0.04008 \\
\hline$p$-Value & 0.00004 & 0.00005 & 0.00004 & 0.00002 & \\
\hline FT-Coffee & $\begin{array}{l}13.20 \\
(2.24)\end{array}$ & $\begin{array}{l}11.75 \\
(1.77)\end{array}$ & $\begin{array}{l}10.71 \\
(0.82)\end{array}$ & $\begin{array}{l}8.835 \\
(0.76)\end{array}$ & 0.00011 \\
\hline OM-Coffee & $\begin{array}{c}9.55 \\
(0.79)\end{array}$ & $\begin{array}{c}9.06 \\
(0.53)\end{array}$ & $\begin{array}{l}9.63 \\
(1.3)\end{array}$ & $\begin{array}{c}8.33 \\
(0.94)\end{array}$ & 0.43406 \\
\hline$p$ value * & 0.00006 & 0.60127 & 0.61638 & 0.43262 & \\
\hline
\end{tabular}

Note: * Tukey's post hoc test; § ANOVA; FT—Filtek; OM-Omnichroma.
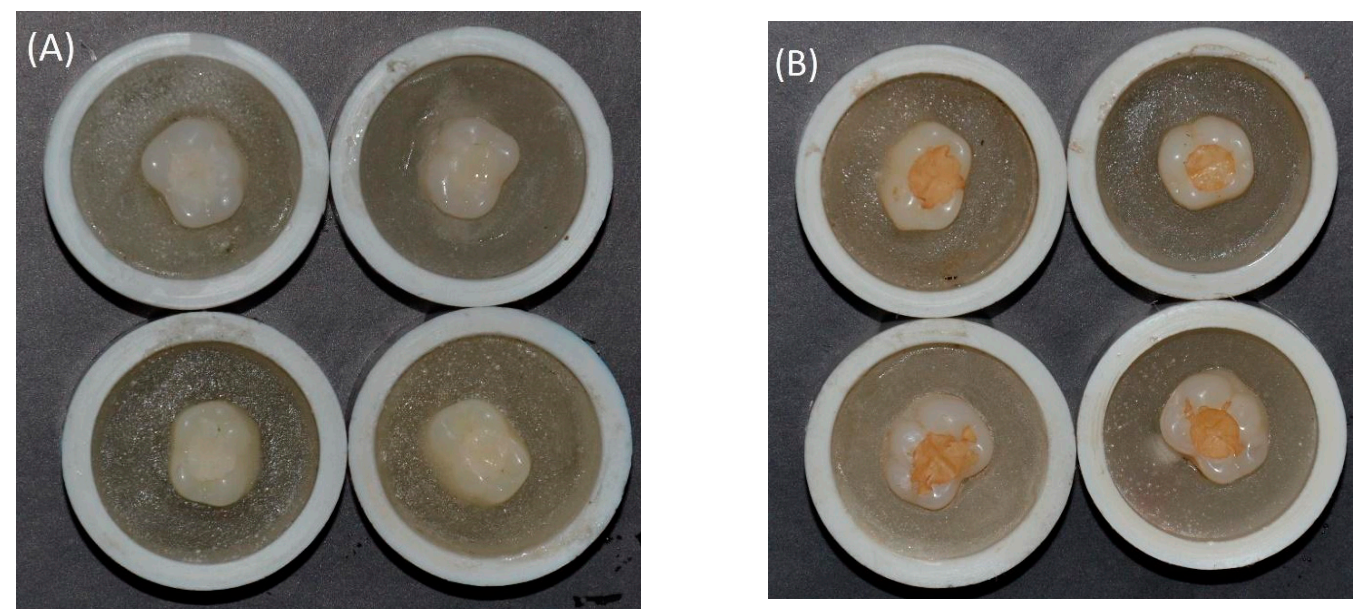

Figure 3. Cont. 

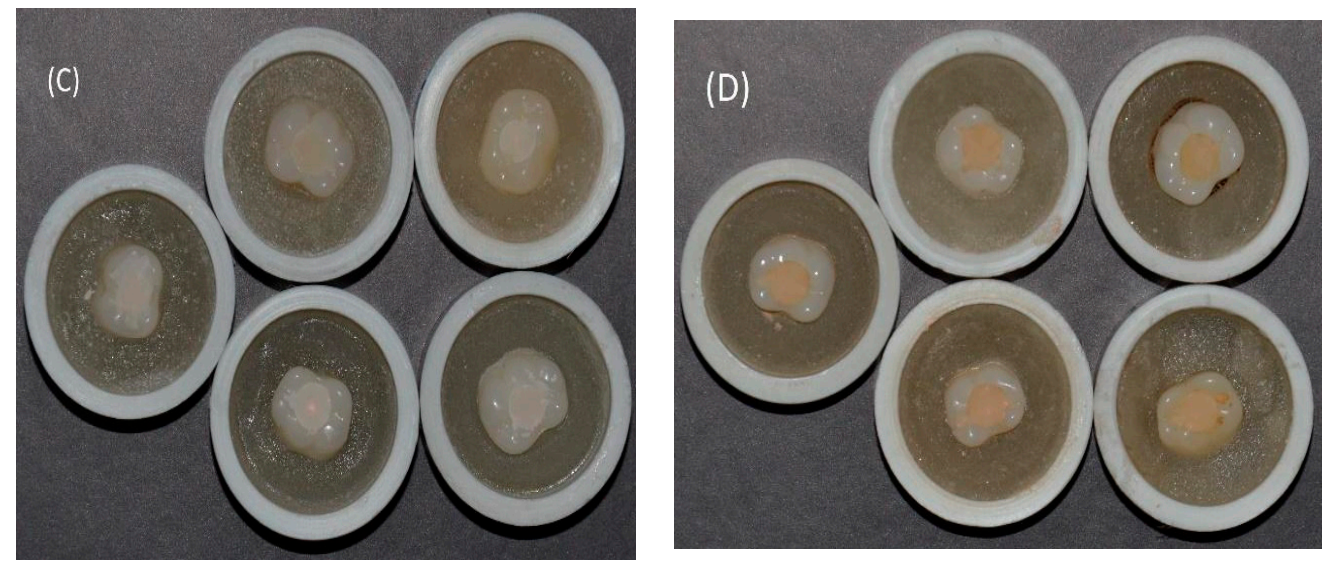

Figure 3. Color stability testing: Filtek specimens after immersion in cola (A) and coffee (B); OM specimens after immersion in cola (C) and coffee (D).

\section{Discussion}

In the present study, the color matching ability of the single-shade resin composite (OM) was compared to the conventional resin composite (FT). Furthermore, the color stability (staining resistance) of the both materials (OM and FT) after cola and coffee immersion was investigated. The outcomes revealed a significantly better color matching ability for conventional resin (FT) for shades B1 and A3 compared to OM resin. However, the color matching ability in shades $\mathrm{B} 2$ and $\mathrm{C} 3$ for $\mathrm{FT}$ and OM resins was comparable. In addition, $\mathrm{OM}$ showed lower color stability (stain resistance) in cola and comparable color stability to the conventional composite in coffee. Therefore, the hypothesis was rejected. A plethora of explanations could be proposed for the outcomes observed regarding color matching and stability.

$\mathrm{OM}$ is considered a smart material with ideal properties to reflect similar shades to those of the surrounding teeth [18]. This material is colorless and the color properties are based on the structural color. According to the current literature, OM comprises a $260 \mathrm{~nm}$ spherical filler, which generates $\mathrm{a}$ and $\mathrm{b}$ color coordinates that are necessary to match natural teeth [18]. Brewer et al. has suggested that the changes in the filler size and composition greatly influence the material's color stability and optical properties [19]. In the present study, the OM filler consisted only of specific, single-sized spherical particles, which enhanced the light reflection within a specific wavelength inside the tooth color space. Therefore, the claims of OM having better control of the optical properties of the material are supported by previous evidence [19].

Moreover, the tooth factor is another variable that influences the color matching ability of the restorative material [19]. The literature states the tooth color depends on the dentin color rather than the enamel color [20]. In previous studies [19,20], enamel showed limited influence on the final shade of dental restorations; however, Saegusa et al. [6] and Oivanen et al. [4] suggested that enamel has a strong influence on color perception in terms of lightness. Moreover, the changes in enamel and dentin for each tooth also greatly influence the efficiency of the restorative material's color stability [11]. In the present study, the authors performed an experiment on acrylic teeth to establish standardization. Denture teeth were used to standardize the color measurements, as it is impossible to standardize the color of natural teeth; however, resin teeth have highly glossed and smooth surfaces (highly polished), which affects color assessments.

Researchers believe that a material's composition greatly influences its optical properties and color matching effects [21]. Among the commonly used material, Filtek presented the highest degree of resistance against discoloration [21]. The hydrophobic nature of Filtek resin causes decreased water sorption, solubility, and diffusion coefficient due to the presence of silorane [22]. The absence of the Bis GMA monomer reduces the hydrophilicity of the material, thereby reducing the risk of saliva absorption, which affects the material 
over a long period. A similar outcome was found in a present study that showed Filtek to have better color stability compared to OM. Recent studies analyzing the color matching ability of OM have suggested the color matching to be $60.8 \%$ [23]. The present study showed similar outcomes, with variable $\Delta \mathrm{E}$ values for $\mathrm{OM}$, the greatest color differences for shade B1, and the smallest differences in shade C3. The possible explanation for the vast differences is the colorless filler powder that strongly reflects light, which enhances the shade's appearance compared to the surrounding structure. However, this structural color phenomenon cannot be displayed on white backgrounds [23]. Moreover, the universal ability to match with each color might be attributed to the high translucency, which aids in light reflection from the wall of the tooth, thereby enabling the tooth to mimic similar shades [23].

Moreover, in the present study, the color stability levels of the composites (FT and $\mathrm{OM}$ ) were measured after specimens had been submerged in cola and coffee for 2 weeks. The restorative composites demonstrated significant differences in cola compared to coffee. FT showed better stain resistance in cola compared to OM, while OM showed comparable stain resistance to FT in coffee. The possible explanation for the color changes is the small filler particles and acidic properties, which erode the surface texture and increase water sorption when teeth are exposed to the fluids, allowing for greater levels of restorative staining, affecting light transmission [24,25]. It has been observed that the $\mathrm{pH}$ of cola ranges between 1.5 and 2, which enables an acceptable $\Delta \mathrm{E}$ change for resin composites [26]. It has also been suggested that the ability of $\mathrm{OM}$ to mimic similar shades to surrounding teeth is due to the filler particles being the same size [26]. In addition, Barbon et al. reported that nanocomposites with smaller particles produce smoother surfaces, reducing surface stains [27]. Furthermore, the lower filler content and the smaller structure of the monomers contributes greatly to the material discoloration, especially under the influence of beverages. A higher filler content directly improves the translucency due to optimal optical scattering of the composite [28]. A study by Mousavinasab [29] showed higher translucency values for composites with high filler contents compared to materials with low filler contents, which also influences the blending effect [30]. However, in the present study, the filler content was not a variable and both resin composites contained similar filler contents; therefore, further studies are warranted to assess the influence of the filler size, shape, and proportions on the color stability of resin composites.

Studies have identified that color changes of less than $3.5 \Delta \mathrm{E}$ are not detectable by the naked eye [30]. In the present study, except for shade B1, all FT and OM-C samples showed color differences of less than $3.5 \Delta \mathrm{E}$. This suggests that although the color changes were higher for OM-C samples than FT samples, the changes were clinically undetectable, suggesting a possible indication of OM-C in clinical patients. Overall, within the experimental limitations, the OM resin composite showed variable color matching and color stability, which was not comparable to the conventional restorative material. The clinical application of in vivo studies is greater than in vitro studies as they demonstrate accurate outcomes based on the impacts of oral conditions. Oral conditions and ageing influence the color stability of resin composites. As the impacts of aging were not assessed in the present study, future studies are recommended to assess the color stability of $\mathrm{OM}$ with ageing. In addition, the surface polish and finish of resin materials impacts their translucency, color stability, and aesthetic outcomes. However, in the present study, the effect of the surface finish was not assessed for the OM single-shade resin. Therefore, further studies investigating the influence of the surface finish, ageing, occlusal loads, and clinical conditions on OM are warranted.

\section{Conclusions}

The color matching ability levels of FT, OM-C, and OM-UC were significantly different. FT showed significantly better color matching in comparison to OM-UC specimens for shades B1, B2, and A3. Shade C3 showed comparable color matching ability for FT and OM-UC. Regarding the color stability in cola, FT samples showed significantly lower $\Delta \mathrm{E}$ 
(better color stability) compared to OM samples for all four shades (B1, B2, A3, C3). The $\triangle \mathrm{E}$ values of $\mathrm{OM}$ and FT samples in coffee were comparable for shades B2, A3, and C3, showing comparable color stability.

The color matching ability of the single-shade resin composite (OM) was influenced by the tooth color. The conventional resin composite showed better color matching ability than the single-shade composite (OM). The color stability of the single-shade resin composite $(\mathrm{OM})$ was dependent on the staining medium. The OM composite showed more compromised color stability than the conventional resin composite in cola and comparable color stability to the conventional resin composite in coffee.

Author Contributions: Conceptualization, E.M.A., S.A.-S., F.A., S.A.-s., and F.V. methodology, A.B., T.A., and K.A.-S., formal analysis, A.A., A.B., F.A., S.A.-S., S.A.-s. and F.V. investigation, A.B., F.A., and F.V.; data curation, E.M.A.,K.A.-S., A.B., F.A., and F.V.; writing-original draft preparation, K.M.A., K.A.-S., A.B., and F.V.; writing-review and editing., E.M.A., S.A.-s., A.B., K.M.A., F.K.A., T.A., K.A.-s., A.B., and F.V.; supervision, F.V., K.M.A., F.K.A., and A.B. All authors have read and agreed to the published version of the manuscript.

Funding: The authors are grateful to the Deanship of Scientific Research, King Saud University, for funding through the Vice Deanship of Scientific Research Chairs, Research Chair for Biological Research in Dental Health.

Institutional Review Board Statement: Not applicable. No human tissue or patients were involved.

Informed Consent Statement: Not applicable. No human tissue or patients were involved.

Data Availability Statement: Data from this study are available on request form the corresponding author.

Conflicts of Interest: Authors declare no conflict of interest.

\section{References}

1. Pereira Sanchez, N.; Powers, J.M.; Paravina, R.D. Instrumental and visual evaluation of the color adjustment potential of resin composites. J. Esthet. Restor. Dent. 2019, 31, 465-470. [CrossRef] [PubMed]

2. de Abreu, J.L.B.; Sampaio, C.S.; Benalcazar Jalkh, E.B.; Hirata, R. Analysis of the color matching of universal resin composites in anterior restorations. J. Esthet. Restor. Dent. 2021, 33, 269-276. [CrossRef] [PubMed]

3. Durand, L.B.; Ruiz-López, J.; Perez, B.G.; Ionescu, A.M.; Carrillo-Pérez, F.; Ghinea, R.; Pérez, M.M. Color, lightness, chroma, hue, and translucency adjustment potential of resin composites using CIEDE2000 color difference formula. J. Esthet. Restor. Dent. 2020, 33, 836-843. [CrossRef] [PubMed]

4. Oivanen, M.; Keulemans, F.; Garoushi, S.; Vallittu, P.K.; Lassila, L. The effect of refractive index of fillers and polymer matrix on translucency and color matching of dental resin composite. Biomater. Investig. Dent. 2021, 8, 48-53. [PubMed]

5. Iyer, R.S.; Babani, V.R.; Yaman, P.; Dennison, J. Color match using instrumental and visual methods for single, group, and multi-shade composite resins. J. Esthet. Restor. Dent. 2021, 33, 394-400. [CrossRef]

6. Saegusa, M.; Kurokawa, H.; Takahashi, N.; Takamizawa, T.; Ishii, R.; Shiratsuchi, K.; Miyazaki, M. Evaluation of Color-matching Ability of a Structural Colored Resin Composite. Oper. Dent. 2020, 46, 306-315. [CrossRef] [PubMed]

7. Lucena, C.; Ruiz-López, J.; Pulgar, R.; Della Bona, A.; Pérez, M.M. Optical behavior of one-shaded resin-based composites. Dent. Mater. 2021, 37, 840-848. [CrossRef] [PubMed]

8. Alkhudhairy, F.; Vohra, F.; Naseem, M.; Owais, M.M.; Amer, A.H.B.; Almutairi, K.B. Color stability and degree of conversion of a novel dibenzoyl germanium derivative containing photo-polymerized resin luting cement. J. Appl. Biomater. Funct. Mater. 2021, 18. [CrossRef]

9. Sharma, N.; Samant, P.S. OMNICHROMA: The See-It-To-Believe-It Technology. EAS J. Dent. Oral Med. 2021, 3, 100-104.

10. Arikawa, H.; Kanie, T.; Fujii, K.; Takahashi, H.; Ban, S. Effect of filler properties in composite resins on light transmittance characteristics and color. Dent. Mater. J. 2007, 26, 38-44. [CrossRef] [PubMed]

11. Alkhudhairy, F.; AlKheraif, A.; Bin-Shuwaish, M.; Al-Johany, S.; Naseem, M.; Vohra, F. Effect of Er, Cr: YSGG laser and ascorbic acid on the bond strength and microleakage of bleached enamel surface. Photomed. Laser Surg. 2018, 36, 431-438. [CrossRef] [PubMed]

12. Evans, M.B. The Visual and Spectrophotometric Effect of External Bleaching on OMNICHROMA Resin Composite and Natural Teeth. Diploma Thesis, West Virginia University, Morgantown, WV, USA, 2020.

13. Vohra, F.; Labban, N.; Al-Hussaini, A.; Al-Jarboua, M.; Zawawi, R.; Alrahlah, A.; Naseem, M. Influence of Er; Cr: YSGG laser on shear bond strength and color stability of lithium disilicate ceramics: An in vitro study. Photobiomodul. Photomed. Laser Surg. 2019, 37, 483-488. [CrossRef] 
14. Peyton, J.H. Restoring Large Class III Cavities with a One-Shade Composite. Available online: https://www.dentaltown. $\mathrm{com} / \mathrm{magazine} /$ article/7843/show-your-work-restoring-large-class-iii-cavities-with-a-one-shade-composite (accessed on 19 August 2021).

15. Almohareb, T.; Alkatheeri, M.S.; Vohra, F.; Alrahlah, A. Influence of experimental staining on the color stability of indirect computer-aided design/computer-aided manufacturing dental provisional materials. Eur. J. Dent. 2018, 12, 269-274. [CrossRef] [PubMed]

16. Gamal, W.M.; Riad, M. Color matching of a single shade structurally colored universal resin composite with the surrounding hard dental tissues. Egypt. Dent. J. 2020, 66, 2721-2727.

17. Chu, S.J.; Trushkowsky, R.D.; Paravina, R.D. Dental color matching instruments and systems. Review of clinical and research aspects. J. Dent. 2010, 38, e2-e16. [CrossRef] [PubMed]

18. Brewer, J.D.; Wee, A.; Seghi, R. Advances in color matching. Dent. Clin. 2004, 48, 341-358. [CrossRef]

19. Perdigão, J.; Araujo, E.; Ramos, R.Q.; Gomes, G.; Pizzolotto, L. Adhesive dentistry: Current concepts and clinical considerations. J. Esthet. Restor. Dent. 2021, 33, 51-68. [CrossRef] [PubMed]

20. Yazdianpour, S. Color Adjustment Potential of Universal Resin Composites in Simulated Class I Restorations. Ph.D. Thesis, The University of Texas School of Dentistry at Houston, Houston, TX, USA, 2021.

21. Hanc1, S.T.; Hancı, Ö.; Yalçın, B.M. Nicotine Addiction and Tooth Loss. Clin. Oral Investig. 2021, 25, 4185-4238.

22. Türker, B.; Koçak, A.; Esra, A. Effect of five staining solutions on the colour stability of two acrylics and three composite resins based provisional restorations. Eur. J. Prosthodont. Restor. Dent. 2006, 14, 2-6.

23. Bayindir, F.; Kürklü, D.; Yanikoğlu, N.D. The effect of staining solutions on the color stability of provisional prosthodontic materials. J. Dent. 2012, 40, e41-e46. [CrossRef] [PubMed]

24. Kumari, R.V.; Nagaraj, H.; Siddaraju, K.; Poluri, R.K. Evaluation of the effect of surface polishing, oral beverages and food colorants on color stability and surface roughness of nanocomposite resins. J. Int. Oral Health 2015, 7, 63. [PubMed]

25. Schroeder, T.; da Silva, P.B.; Basso, G.R.; Franco, M.C.; Maske, T.T.; Cenci, M.S. Factors affecting the color stability and staining of esthetic restorations. Odontology 2019, 107, 507-512. [CrossRef] [PubMed]

26. Pruthi, G.; Jain, V.; Kandpal, H.C.; Mathur, V.P.; Shah, N. Effect of bleaching on color change and surface topography of composite restorations. Int. J. Dent. 2010, 2010, 695748. [CrossRef]

27. Barbon, F.J.; Moraes, R.R.; Calza, J.V.; Perroni, A.P.; Spazzin, A.O.; Boscato, N. Inorganic filler content of resin-based luting agents and the color of ceramic veneers. Braz. Oral Res. 2018, 32, e49. [CrossRef]

28. Son, S.A.; Park, J.K.; Seo, D.G.; Ko CCKwon, Y.H. How light attenuation and filler content affect the microhardness and polymerization shrinkage and translucency of bulk-fill composites? Clin. Oral Investig. 2017, 21, 559-565. [CrossRef] [PubMed]

29. Mousavinasab, S.M. Effects of filler content on mechanical and optical properties of dental composite resin. In Metal, Ceramic and Polymeric Composites for Various Uses, 1st ed.; In Tech.: Rijeka, Croatia, 2011; pp. 421-428.

30. Mizutani, K.; Ishii, R.; Takamizawa, T.; Shibasaki, S.; Kurokawa, H.; Suzuki, M.; Tsujimoto, A.; Miyazaki, M. Flexural Properties and Polished Surface Characteristics of a Structural Colored Resin Composite. Oper. Dent. 2021, 46, E117-E131. [CrossRef] [PubMed] 